\title{
Automatic selection for general surrogate models
}

\author{
Malek Ben Salem · Lionel Tomaso
}

Received: date / Accepted: date

\begin{abstract}
In design engineering problems, the use of surrogate models (also called metamodels) instead of expensive simulations have become very popular. Surrogate models include individual models (regression, kriging, neural network...) or a combination of individual models often called aggregation or ensemble. Since different surrogate types with various tunings are available, users often struggle to choose the most suitable one for a given problem. Thus, there is a great interest in automatic selection algorithms. In this paper, we introduce a universal criterion that can be applied to any type of surrogate models. It is composed of three complementary components measuring the quality of general surrogate models: internal accuracy (on design points), predictive performance (cross-validation) and a roughness penalty. Based on this criterion, we propose two automatic selection algorithms. The first selection scheme finds the optimal ensemble of a set of given surrogate models. The second selection scheme further explores the space of surrogate models by using an evolutionary algorithm where each individual is a surrogate model. Finally, the performances of the algorithms are illustrated on 15 classical test functions and compared to different individual surrogate models. The results show the efficiency of our approach. In particular, we
\end{abstract}

Malek BEN SALEM is funded by a CIFRE grant from the ANSYS company, subsidized by the French National Association for Research and Technology (ANRT, CIFRE grant number 2014/1349).

\section{Ben Salem}

Ecole des mines de St-Etienne - ANSYS, Inc. Villeurbanne

Tel.: $+33-\ldots$

Fax.: $+33-\ldots$

E-mail: firstname.ben-salem[@]emse.fr

L. Tomaso

ANSYS, Inc. Villeurbanne

Tel.: $+33-\ldots$

Fax.: $+33-.$.

E-mail: firstname.name[@]ansys.com observe that the three components of the proposed criterion act all together to improve accuracy and limit over-fitting.

Keywords Surrogate modeling - Multiple Surrogate models · Surrogate Model selection · Cross-Validation errors

\section{PACS PACS fx $122 \cdot$ PACS fx $122 \cdot$ MSC 4563}

\section{Introduction}

Computer simulations are an efficient tool to study complex physical behaviors. However, high-fidelity simulations are generally computationally expensive. Therefore, surrogate models, also known as metamodels or response surfaces, are usually instead used. They provide an approximation of a response of interest based on a limited number of expensive simulations. There are several methods of construction of such approximations. Among the popular surrogate model types, we can cite for example Kriging (Matheron 1963), support vector machines (SVM) (Smola and Schlkopf. 2004), Moving least squares (Lancaster and Salkauskas, 1981) and Multivariate Adaptive Regressive Splines (MARS) (Friedman, 1991). Generally, a metamodel family comes with several possible tunings. In the same time, there is no universal optimal surrogate for all the problems. Some users face some difficulties in selecting the most suitable surrogate for their problem. Thus, there is a great interest in automatic model selection algorithms. The main purpose is to choose the surrogate that provides the best prediction performances on the whole parametric space.

In the literature, this problem is generally studied along three different approaches.

1) The first approach consists in using algorithms to optimize the settings of a particular surrogate model type. For instance, (Chen et al, 2004; Lessmann et al, 2006) 
work on SVM, (Zhang et al, 2000) on neural networks, and (Tomioka et al, 2007) deal with least squares regression.

2) A second approach consists in considering multiple surrogates or ensembles. The automatic surrogate selection is so a model selection method. Often, the selected model is a weighted sum of different surrogate models. For example, (Viana et al, 2009, Zhou et al, 2011; Acar and Rais-Rohani, 2009, Goel et al, 2007) discuss different ways to build such aggregations.

3) The last approach consists in selecting a good member among different types of surrogate models with different settings. We refer for instance to the works of (Gorissen et al, 2009; Shi et al, 2012, Zhou and Jiang, 2016).

The main objective of our paper is to propose a new relevant surrogate model selection algorithm that can handle different type of surrogates. To achieve such a goal, we define a universal criterion. This criterion may evaluate the accuracy of any surrogate model.

The paper is organized as follows. We introduce and discuss in Section 2 our criterion called the Penalized Predictive Score $(P P S)$. We show in Section 3 that PPS is suitable to optimize weights of surrogate models ensembles. In Section 4. we present an evolutionary selection algorithm that explores the space of surrogate models. The algorithm is called PPS Genetic Aggregations (PPS-GA). Finally, the performances of the algorithm on 15 test cases are displayed in Section 5 The results show the efficiency of the PPS, the complementary role of its three components and the relevance of the proposed selection algorithms.

\section{Penalized Predictive Score (PPS)}

\subsection{Definition}

Assessing the quality of a surrogate is very challenging. It is desirable to use an independent set to assess the predictive capabilities of a given method. But, this is computationally expensive in practice. One can also estimate the errors by computing the errors on design points. Unfortunately, a small MSE does not imply good predictive capabilities. Therefore, resampling techniques such as Cross-Validation (CV) (Stone, 1974) or bootstrap (Efron and Tibshirani, 1993) are generally used. Such techniques reduce the bias of the estimation. Nevertheless, they does not prevent overparameterized models. We will introduce a criterion that will do this job. This criterion is called the Penalized Predictive Score (PPS Equation (1)). It combines three components:

a) The internal accuracy (or fit): we use the mean squared errors (MSE) on design points.

b) The predictive capability: we propose to use the $10 \mathrm{~F}-\mathrm{CV}$ PRESS errors. c) A roughness penalty: We propose to use the Bending Energy Functional (BEF) ((Duchon, 1977)).

$\operatorname{PPS}\left(m, \mathbf{Z}_{\mathbf{n}}\right)=\underbrace{\alpha \widehat{\mathscr{R}}_{l_{2}, \mathbf{Z}_{\mathbf{n}}}(m)}_{a}+\underbrace{\beta \mathscr{R}_{10-C V}(m)}_{b}+\underbrace{\gamma E_{n}(m)}_{c}$

Here, as it will be described below, $\widehat{\mathscr{R}}_{l_{2}, \mathbf{Z}_{\mathbf{n}}}(m)$ denotes the MSE criterion, $\mathscr{R}_{10-C V}(m)$ the 10 -Fold cross-validation estimate of the errors and $E_{n}(m)$ a roughness penalty. Further, $\alpha, \beta, \gamma$ are weights in $\mathbb{R}_{+}$. In all our implementations, we use $\alpha=2 \beta$ and $\beta=2 \gamma$.

\subsection{Internal accuracy}

Let $\Omega=[0,1]^{d}$ be the parametric space of dimension $d$. $\mathbf{X}_{\mathbf{n}}=\left(\mathbf{x}_{\mathbf{1}}, \ldots, \mathbf{x}_{\mathbf{n}}\right)^{\top} \in \Omega^{n}$ and $\mathbf{Y}_{\mathbf{n}}=\left(y_{1}, \ldots, y_{n}\right)^{\top} \in \mathbb{R}^{n}$ form the set of design points $\mathbf{Z}_{\mathbf{n}}=\left(\mathbf{X}_{\mathbf{n}}, \mathbf{Y}_{\mathbf{n}}\right)$ where $y_{i}=f\left(\mathbf{x}_{\mathbf{i}}\right)$ for $i=1, \ldots, n$ and $f \in \mathbb{R}^{\Omega}$ is an expensive-to-evaluate function. A surrogate model $\widehat{m}_{\mid \mathbf{Z}_{\mathbf{n}}} \in \Omega^{\mathbb{R}}$ is used to replace $f$ based on the design $\mathbf{Z}_{\mathbf{n}}$. We call the construction method a "surrogate model builder". For instance, if $m$ is a surrogate model builder, then we build the surrogate model $\widehat{m}_{\mid \mathbf{Z}_{\mathbf{n}}} \in \Omega^{\mathbb{R}}$ based on the design $\mathbf{Z}_{\mathbf{n}}$.

The assessment of the performance of a surrogate model is extremely important in practice (Hastie et al, 2009). It relies on the evaluation on the set of design points of the prediction capabilities of the surrogate model. It is generally based on a contrast function (or loss function) that measures the errors between the predicted and the true models. A typical choice is the square error $l_{2}(x, y)=(x-y)^{2}$. The integral form of the MSE is the $l_{2}-$ risk overall the parametric space.

$\mathscr{R}_{l_{2}, \mathbf{Z}_{\mathbf{n}}}(m)=\int_{\Omega} l_{2}\left(\widehat{m_{\mid \mathbf{Z}_{\mathbf{n}}}}(\mathbf{x}), f(\mathbf{x})\right) d \mathbf{x}$

Since $f$ is unknown, we can only use an approximation to estimate this risk. Ideally, the performance of the surrogate model would be evaluated on an extra set of points. However, generating such set is sometimes computationally expensive. Therefore, one use an empirical distribution associated to the set of design points. Computing the mean square errors (MSE) (Equation (3)) on the set of design points for the surrogate model $\widehat{m_{\mid \mathbf{Z}_{\mathbf{n}}}}$ is an empirical approximation of $\mathscr{R}_{l_{2}, \mathbf{Z}_{\mathbf{n}}}(m)$ defined in Equation (2).

$$
\begin{aligned}
\widehat{\mathscr{R}}_{l_{2}, \mathbf{Z}_{\mathbf{n}}}(m) & =\frac{1}{n} \sum_{i=1}^{n} l_{2}\left(\widehat{m_{\mid \mathbf{Z}_{\mathbf{n}}}}\left(x_{i}\right), y_{i}\right) \\
& =\frac{1}{n} \sum_{i=1}^{n}\left(\widehat{m_{\mid \mathbf{Z}_{\mathbf{n}}}}\left(x_{i}\right)-y_{i}\right)^{2}
\end{aligned}
$$

Note that computing the MSE on the set of design points is a biased estimate of the error in the whole space. In fact, 
for any interpolating surrogate model $m, \widehat{\mathscr{R}}_{l_{2}, \mathbf{Z}_{\mathbf{n}}}(m)=0$. This does not necessarily mean that the surrogate model fits the real function in the whole space.

\subsection{Predictive capabilities}

On one hand, the use of design points to estimate the errors yields an optimistic result (Arlot and Celisse, 2010). On the other hand, using a validation set can be expensive. Therefore, it is convenient to use re-sampling techniques such as Cross-Validation (CV) (Stone, 1974) and bootstrap (Efron and Tibshirani, 1993) to estimate the predicted errors. Resampling techniques estimate the errors by using subsets of the design points to build several sub-surrogate models. For instance, computing the Leave-One-Out CrossValidation (LOO-CV) errors of a surrogate model $\widehat{m_{\mid \mathbf{Z}_{\mathbf{n}}}}$ consists in computing the errors of an observation $\left(\mathbf{x}_{\mathbf{i}}, y_{i}\right)$ based on the surrogate model $\widehat{m_{\mid \mathbf{Z}_{\mathbf{n},-\mathbf{i}}}}$ built on the subset of all the design points expect the $i^{\text {th }}$ design point $\left(\mathbf{Z}_{\mathbf{n},-i}=\left(\mathbf{x}_{\mathbf{j}}, \mathbf{y}_{\mathbf{j}}\right)_{j \neq i}\right)$. In the same way, $k$-fold cross-validation $(k \mathrm{~F}-\mathrm{CV})$ consists in dividing the data into $k$ subsets. Each subset plays the role of validation set while the remaining $k-1$ subsets are used together as the training set. If $k$ is the number of folds, for $i \in 1, \ldots, k$ let $\mathbf{Z}^{(i)} \in \mathscr{P}\left(Z_{n}\right)$ be a subset of $Z_{n}$ such that $\cup_{i=1}^{k} \mathbf{Z}^{(i)}=Z_{n}$. The $k \mathrm{~F}-\mathrm{CV}$ estimates of the $l_{2}$ errors (Equation (4)) by computing the loss of a point in the $i^{\text {th }}$ fold $\mathbf{Z}^{(i)}$ compared to the prediction of the surrogate model built on the remaining folds $\left(\mathbf{Z}_{n} \backslash \mathbf{Z}^{(i)}\right)$.

$\mathscr{R}_{k-C V}(m)=\frac{1}{n} \sum_{i=1}^{k} \sum_{\left(x^{\prime}, y^{\prime}\right) \in \mathbf{Z}^{(i)}} l_{2}\left(\widehat{m_{\mid \mathbf{Z}_{n} \backslash \mathbf{Z}^{(i)}}}\left(x^{\prime}\right), y^{\prime}\right)$

where $\mathbf{z} \in \mathbf{Z}_{n} \backslash \mathbf{Z}^{(i)}$ if and only if $\mathbf{x} \in \mathbf{Z}_{n}$ and $\mathbf{x} \notin \mathbf{Z}^{(i)}$.

(Queipo et al, 2005) pointed out that the main advantage of $\mathrm{CV}$ is that it provides a nearly unbiased estimate. Further, (Kohavi, 1995) studied Cross-Validation and Bootstrap performances on a large dataset and recommended using stratified 10-fold-cross-validation. (James et al, 2013) stated that $k \mathrm{~F}-\mathrm{CV}$ with $k=5$ or $k=10$ yield test error estimates that suffer neither from excessively high bias nor from very high variance.

\subsection{Penalization}

Penalties are used in several model selection frameworks in order to prevent over-fitting. Selection criteria such as the Bayesian Information Criterion (BIC) (Schwarz et al, 1978) or Akaike Information Criterion (AIC) (Akaike, 1974) penalize the models by their degrees of freedom. Most penalties are designed for a particular family of surrogates. Here, we are interested in universal methods. So that, we prefer to deal with the smoothness of the surrogate model rather than with its structural complexity. For instance, (Nguyen et al 2011) introduce a criterion called Linear Reference Model (LRM). It scores a surrogate model by computing the deviation between its predictions and a local linear model $\widehat{l_{r m}}$. The LRM is computed over a set of $N$ points $x^{(k)}$ for $k=1, \ldots, N$ (see Equation (5)).

$$
\mathscr{R}_{L R M}(m)=\frac{1}{N} \sum_{k=1}^{N} l_{2}\left(\widehat{m_{\mid \mathbf{Z}_{\mathbf{n}}}}\left(x^{(k)}\right), \widehat{l_{r m}}\left(x^{(k)}\right)\right)
$$

Computationally, this last criteria needs the construction of a Delaunay tessellation (Watson 1981) to compute $\widehat{l_{r m}}$. The computational cost of such construction in high dimension is too expensive. We suggest to use a criterion that penalize the roughness of surrogate models: the thin plate spline (TPS) (Duchon, 1977) Bending Energy Functional (BEF). It is a second order partial derivatives-based penalty. For a dimension $d$, the roughness penalty $E_{n}$ is the integral of the squared term of the Hessian (Equation (6)).

$E_{n}(\hat{f})=\int_{\Omega} \sum_{i=1}^{d} \sum_{j=1}^{d}\left(\frac{\partial^{2} \hat{f}}{\partial x_{i} \partial x_{j}}\right)^{2} d x$

LRM can be used in place of the BEF in the selection criterion PPS. It penalizes the deviation from a linear model regardless of its roughness. It still gives good predictive capabilities also. Nevertheless, some rough surrogates may be selected.

\section{Surrogate model ensemble: $P P S-O S$}

\subsection{Overview}

Surrogate model selection consists in selecting a surrogate model among a collection of them. This means that we evaluate the performances of several surrogate models and then choose one of them. (Acar and Rais-Rohani, 2009) stated that this practice has some shortcomings as it does not take full advantage of the resources devoted to constructing different metamodels. In fact, it is possible to consider a weighted combination of surrogates without any significant extra computational cost. These combinations are called: ensembles, aggregations and multiple surrogates.

(Forrester and Keane, 2009) show that these aggregation methods drastically improve the performances of the surrogate models. In general, ensembles require small computational resources compared to the cost of the simulations (Queipo et al, 2005). The general form of an aggregation of 
$p$ surrogate models $\widehat{m^{(i)}} \mid \mathbf{Z}_{\mathbf{n}}$, for $i=1, \ldots, p$ is given in Equation (7):

$\widehat{A}_{\mid \mathbf{Z}_{\mathbf{n}}}(x)=\sum_{i=0}^{p} w_{i}(x) \widehat{m^{(i)}} \mid \mathbf{Z}_{\mathbf{n}}(x)$

For instance, (Zerpa et al, 2005) considered a local combination called weighted average model where the weights are based on the local expected variances of the surrogate models. (Goel et al, 2007) extended the use of ensembles to the identification of region with high error. They presented also several heuristics to weight ensembles.

However, (Gorissen et al, 2009) used a simple average ensemble (all the weights are equal). (Müller and Piché, 2011) proposed to weight the aggregation using the DempsterShafer theory where the error estimates are used as basic probability assignments. (Viana et al, 2009) proposed to use an ensemble of surrogate models that minimize the CV errors. In fact, if for $k=1, \ldots, n, \mathbf{v}_{\mathbf{k}}$ is the vector of CV errors of the surrogate model $\widehat{m^{(i)}} \mid \mathbf{Z}_{\mathbf{n}}$, the CV errors of the aggregation is then $W^{\top} C W$. The weights are selected to minimize the $\mathrm{CV}$ errors of the aggregation under the constraint $\sum_{i=1}^{p} w_{i}=1$. The optimal weighted surrogate $O W S$ is obtained using the weights of Equation (8).

$\mathbf{W}=\frac{\mathbf{C}^{-1} \mathbf{1}}{\mathbf{1}^{\top} \mathbf{C}^{-1} \mathbf{1}}$

where the elements of the matrix $\mathbf{C}, c_{i j}=<\mathbf{v}_{\mathbf{i}}, \mathbf{v}_{\mathbf{j}}>$. (Viana et al, 2009) noticed that the solution may include negative values. They stated that this additional freedom to the weights estimation amplify errors. In fact, the matrix $\mathbf{C}$ is an approximation of the covariance of the errors of the surrogate models. To overcome the problem, the authors suggested to use only the diagonal elements of $\mathbf{C}$. Then, the weights are $w_{i}=\frac{c_{i i}^{-1}}{\sum_{k=1}^{n} c_{k k}^{-1}}$. This formulation is close to the weights of the PRESS weighted surrogate $(P W S)$ given in (Goel et al, 2007) (equation (9), with $\alpha=0, \beta=-2$.

$w_{i}=\frac{\left(\sqrt{c_{i i}}+\frac{\alpha}{n} \sum_{j=1}^{n} \sqrt{c_{j j}}\right)^{\beta}}{\sum_{k=1}^{n}\left(\sqrt{c_{k k}}+\frac{\alpha}{n} \sum_{j=1}^{n} \sqrt{c_{j j}}\right)^{\beta}}$

\subsection{PPS-optimal ensemble}

Let us consider $\left.\widehat{\left(m^{(1)}\right.}\left|\mathbf{Z}_{\mathbf{n}}, \ldots, \widehat{m^{(n)}}\right| \mathbf{Z}_{\mathbf{n}}\right)$ a set of $p$ surrogate models. Let $A$ be an aggregation of these surrogate models weighted by the vector $\mathbf{W}=\left(w_{1}, . ., w_{n}\right)$ (Equation $[10)$.

$\widehat{A}(x)=\sum_{k=1}^{p} w_{k} \widehat{m^{(k)}} \mid \mathbf{Z}_{\mathbf{n}}(x)$

In our formulation, we compute the weights of the aggregations by optimizing the PPS of the aggregation under the constraint $\sum_{i=1}^{p} w_{i}=1$. The PPS-Optimal aggregation is then the aggregation in which the weights are the solution of the optimization Problem (11).

$$
\begin{array}{ll}
\min _{W} & \operatorname{PPS}\left(A, \mathbf{Z}_{\mathbf{n}}\right) \\
\text { u.c. } & \sum_{i=1}^{p} w_{i}=1
\end{array}
$$

For each $k$ in $1, \ldots, p$, let:

- $\mathbf{e}_{\mathbf{k}}$ be the vector of errors on design points.

- $\mathbf{v}_{\mathbf{k}}$ the vector of cross-validation error of the surrogate $\left.\operatorname{model} \widehat{m^{(k)}}\right)_{\mid \mathbf{Z}_{\mathbf{n}}}$.

Notice then that the MSE of the aggregation is a quadratic form of the weights

$$
\mathscr{R}_{l_{2}, \widehat{P}_{n}}(A)=\left\|\sum_{i=1}^{p} w_{i} e_{i}\right\|=\mathbf{W}^{T} \mathbf{E W}
$$

Where the elements of $\mathbf{E}, E_{i j}=<e_{i}, e_{j}>$. Similarly, the cross validation errors of the aggregation is also a quadratic form of the weights (Equation (13)) where $\mathbf{C}$ is the same defined in the previous section.

$\mathscr{R}_{C V}(A)=\mathbf{W}^{T} \mathbf{C W}$

Last, the energy functional is also a quadratic form of the weights (Equation 14).

$$
\begin{aligned}
E_{n}(\widehat{A}) & =\int_{\Omega} \sum_{i=1}^{d} \sum_{j=1}^{d}\left(\frac{\sum_{k=1}^{p} w_{k} \partial^{2} \widehat{m^{(k)}} \mid \mathbf{Z}_{\mathbf{n}}(x)}{\partial x_{i} \partial x_{j}}\right)^{2} d x \\
& =\mathbf{W}^{T} \mathbf{K W}
\end{aligned}
$$

where: $\mathbf{K}=\left[k_{k l}=\sum_{i=1}^{d} \sum_{j=1}^{d} \int_{\Omega}\left(\frac{\partial^{2} \widehat{m^{(k)}} \mid \mathbf{Z}_{\mathbf{n}}(x)}{\partial x_{i} \partial x_{j}}\right)\left(\frac{\partial^{2} \widehat{m^{(l)}} \mid \mathbf{Z}_{\mathbf{n}}(x)}{\partial x_{i} \partial x_{j}}\right) d x\right]$.

Let $\mathbf{R}=\alpha \mathbf{E}+\beta \mathbf{C}+\gamma \mathbf{K}$. The PPS of the aggregation is then a quadratic form of the weights $\mathbf{W}: P P S(\widehat{A})=\mathbf{W}^{T} \mathbf{R W}$. The PPS-Optimal aggregation is then the aggregation that minimizes the PPS under the constraint $\sum_{i=1}^{n} w_{i}=1$. The solution is defined in Equation (15):

$\mathbf{W}^{\star}=\frac{\mathbf{R}^{-1} \mathbf{1}}{\mathbf{1}^{\top} \mathbf{R}^{-1} \mathbf{1}}$ 


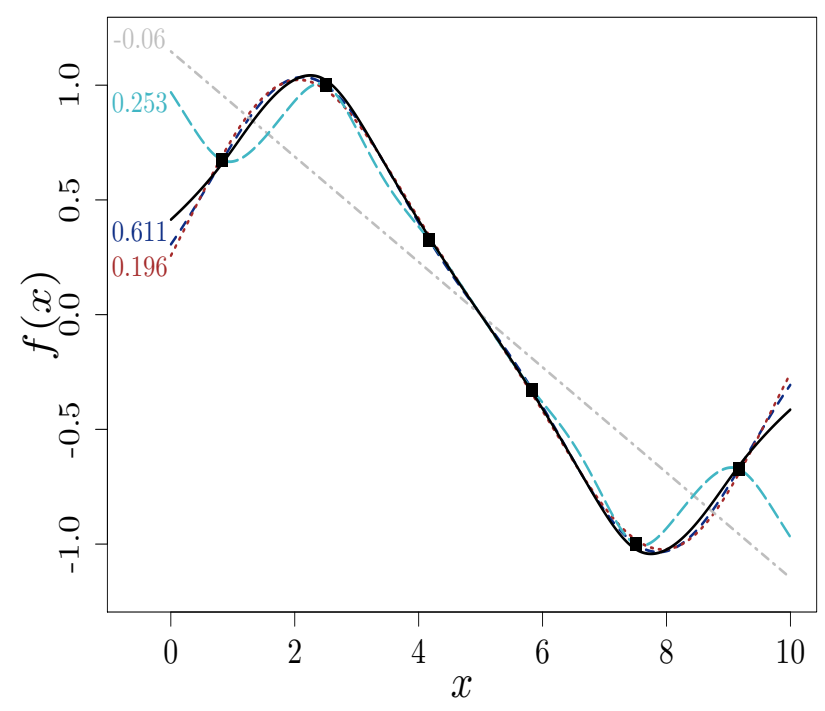

Fig. 1: Example of PPS-Optimal ensemble, Dashed lines: 4 meta-models predictions. Solid line: PPS-optimal ensemble predictions. Black squares: design points

Similarly to Equation (8), the solution of Equation (15) may include negative weights as well as weights greater than one. Unlike, in (Viana et al, 2009) in which the writers suggested to use only the diagonal terms in the matrix to ensure the positivity, here we tolerate such weights since this freedom is controlled by the BEF penalization. As a matter of fact, the BEF penalization prevents to artificial oscillations on the aggregated surrogate.

\subsection{Illustrative example}

We consider the example in Figure 1 The ensemble is the optimal trade-off defined by the PPS parameters. The ensemble is relatively smoother than the interpolating ones of the initial collection. Further, its CV error is lesser than the best prediction of this collection.

\subsection{One shot metamodel selection: PPS-OS}

We suppose that we have at hands $p$ possible surrogate model builders where $p$ is relatively small (typically $p \leq 35$ ). One select the model that has the best PPS. In order to improve the result, we select the PPS-Optimal ensemble. We consider this procedure (described in Algorithm 3.1) as a model selection algorithm. Notice that the aggregation does not increase significantly the computational cost of the procedure as the errors have been generally previously evaluated. $\overline{\text { Algorithm 3.1 PPS One Shot (PPS-OS) model selection al- }}$ gorithm

Inputs: Design Points $\mathbf{Z}_{\mathbf{n}}$

Generate the list of first population of surrogate models builder $L=\left(m_{1}, m_{2}, \ldots, m_{p}\right)$.

Compute the PPS-Optimal aggregation $\widehat{A}$

Outputs: $\widehat{A}$

In our implementation, $P P S$-OS selects the PPS-optimal aggregation of 32 surrogate models from 4 different surrogate types (Kriging, SVM, Polynomial regression and MLS).

\section{PPS-based Genetic Aggregation for model selection : (PPS-GA)}

As discussed in the previous section, the use of PPS to perform model selection is straightforward if the number of the available surrogate model is moderate. In that case, one can consider a weighted PPS-Optimal aggregation of all the possible surrogate models. However, there are many types of surrogate models and each type has several possible settings. For instance, to tune a universal kriging surrogate model, there are various possible choices for covariance function and trend function. Consequently, one cannot evaluate the PPS for all the possible combinations. Even with a good selection criterion, one need to explore the space of available surrogate models to select the best one.

(Gorissen et al, 2009) proposed an evolutionary algorithm to perform surrogate model selection and to explore the space of surrogate models. The surrogate models are considered as the individuals of the population. The settings of the surrogate models are considered as the genetic information of the individuals. The mutation and cross-over operators between two surrogate models of the same type are performed by modifying or exchanging the surrogate models settings. Further, they generate an equally weighted surrogate model ensemble when the cross-over is between two surrogate models of different types. Their algorithm uses the island model of evolutionary algorithms.

We now introduce our selection algorithm based on the genetic aggregation called PPS-GA. Similarly to (Gorissen et al, 2009)'s heuristic, the mutation and cross-over operators are performed over surrogate model builders settings. In our algorithm, all the aggregation weights are now optimized according to the PPS. Moreover, we add new aggregations at each iteration. The members of these aggregations are generated randomly. Further, we do not adopt the island model. We consider that the heterogeneous set of surrogate model builders "lives" together in the same space. The selection method is designed to conserve the diversity. 


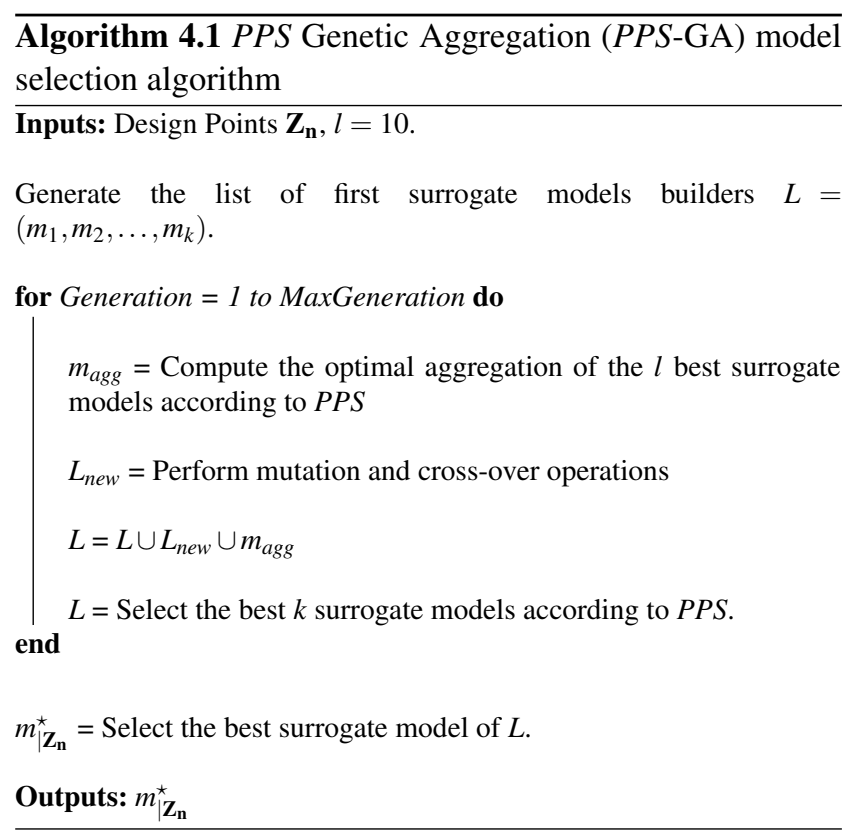

In our implementation, we consider several surrogate types with various settings: Kriging, moving least squares, polynomial regression and support vector machines regression. PPS-GA has another interesting property. It is easy to enrich the set of surrogate model builders. In fact, the algorithm does not require any particular assumption. It is in part due to the universality of $P P S$.

\section{Numerical examples}

\subsection{Benchmark problems}

In order to check the efficiency of PPS-OS and PPS-GA, we tested their performances on a benchmark of 15 func- tions (see Table 1 and formula given in Appendix. B). For each function, we generated 10 different optimized maximin Latin hypercube sampling (LHS) (McKay et al, 1979) of size $N$. We generated an extra test set of size $n_{t}=1000 \times N$ by a fast optimized LHS algorithm (Viana et al, 2010). We use the RMSE criterion (Equation (16)) to evaluate the performances on the set of verification points.

$R M S E=\sqrt{\frac{1}{n_{t}} \sum_{i=1}^{n_{t}}\left(y_{i}-\widehat{y_{i}}\right)^{2}}$

For each function, we compare the performance of the selection algorithms (PPS-OS and PPS-GA) to the performances of 4 witness surrogate models:

a) A kriging surrogate model using an an-isotropic Matérn $5 / 2$ kernel and a linear trend function.

b) A support vector regression using a Gaussian kernel and $\varepsilon$-regression paradigm.

c) A moving least squares surrogate model using a Gaussian weighting function and second order polynomial regression.

d) Full second order polynomial regression, we use leastnorm when the equation system is undetermined.

These surrogates are selected among the 32 surrogates of PPS - OS as follows: We consider the 150 functions (15 $\times 10$ repetitions). For each surrogate $\widehat{m}$, we compute $N_{\text {best }}(\widehat{m})$ : the number of times where $\widehat{m}$ is the best individual surrogate. Each witness surrogate models is the one with highest $N_{\text {best }}$ among its type. The surrogate with the highest $N_{\text {best }}$ is the kriging using an an-isotropic Matérn 5/2 kernel and a linear trend function. It is the best individual surrogate in 25 test $(16 \%)$.

Table 1: Test functions

\begin{tabular}{llll}
\hline Name & Dimension $d$ & Number of design points & Number of test points $n_{t}$ \\
& & 45 & 45000 \\
1. Wing weight function & 10 & 40 & 40000 \\
2. Borehole function & 8 & 75 & 75000 \\
3. Dette \& Pepelyshev (8-Dim) function & 8 & 60 & 60000 \\
4. Piston simulation function & 7 & 35 & 35000 \\
5. OTL circuit function & 6 & 85 & 85000 \\
6. Gramacy \& Lee (2009) function & 6 & 35 & 35000 \\
7. Friedman function & 5 & 16 & 16000 \\
8. Dette \& Pepelyshev exponential function & 3 & 18 & 18000 \\
9. Dette \& Pepelyshev curved function & 3 & 20 & 12000 \\
10. Lim non-polynomial function & 2 & 10 & 20000 \\
11. Currin exponential function & 2 & 45 & 10000 \\
12. Franke's function & 2 & 10 & 45000 \\
13. Gramacy \& Lee (2008) function & 2 & 15 & 10000 \\
14. Sasena function & 2 & 1 & 15000 \\
15. Gramacy \& Lee (2012) function & & & \\
\hline
\end{tabular}




\subsection{Results}

We display the results of the benchmark in Table 2 and in Figures 2, 16 .

- In Table 2, the median and the standard deviation of the RMSE of each surrogate model are given. The best median value is in bold.

- In Figures 2 16, the box-plots illustrate the variability with respect to the design set.
The results show the efficiency of the selection algorithms: the models selected by PPS-OS and PPS-GA outperform each individual surrogate models in the predictive capabilities for at least one function. Generally, the RMSE of the selected surrogates is generally either the best or close to the best one.

\begin{tabular}{|c|c|c|c|c|c|c|c|c|c|c|c|c|}
\hline & \multicolumn{2}{|c|}{ MLS } & \multicolumn{2}{|c|}{ SVM } & \multicolumn{2}{|c|}{ Poly } & \multicolumn{2}{|c|}{ Kriging } & \multicolumn{2}{|c|}{$P P S$-OS } & \multicolumn{2}{|c|}{$P P S$-GA } \\
\hline & mean & $\mathrm{sd}$ & mean & $\mathrm{sd}$ & mean & $\mathrm{sd}$ & mean & $\mathrm{sd}$ & mean & $\mathrm{sd}$ & mean & $\mathrm{sd}$ \\
\hline Wing Weight & 6.646 & 0.500 & 12.889 & 0.225 & 15.890 & 4.332 & 5.800 & 1.076 & 3.873 & 0.708 & 3.701 & 0.560 \\
\hline Borehole & 12.077 & 1.933 & 13.267 & 0.442 & 1341.448 & 2050.563 & 9.014 & 2.128 & 3.197 & 0.418 & 3.627 & 0.467 \\
\hline Dette \& Pepelyshev 8-Dim & 14.574 & 11.524 & 5.236 & 0.134 & 10.819 & 9.006 & 1.771 & 0.780 & 1.995 & 0.902 & 3.609 & 0.162 \\
\hline Piston Simulation & 0.037 & 0.002 & 0.040 & 0.001 & 0.087 & 0.083 & 0.016 & 0.006 & 0.011 & 0.001 & 0.014 & 0.003 \\
\hline OTL Circuit & 0.287 & 0.141 & 0.312 & 0.004 & 0.303 & 0.172 & 0.112 & 0.037 & 0.036 & 0.011 & 0.055 & 0.013 \\
\hline Gramacy \& Lee 2009 & 1.421 & 0.498 & 0.669 & 0.012 & 1.223 & 0.667 & 0.410 & 0.092 & 0.243 & 0.139 & 0.380 & 0.179 \\
\hline Friedman & 4.215 & 1.607 & 1.522 & 0.107 & 4.218 & 1.714 & 1.251 & 0.244 & 0.634 & 0.284 & 0.854 & 0.195 \\
\hline Dette \& Pepelyshev Exp & 0.955 & 0.038 & 2.860 & 0.147 & 0.998 & 0.032 & 3.280 & 0.175 & 1.139 & 0.362 & 1.293 & 0.665 \\
\hline Dette \& Pepelyshev Curved & 1.765 & 0.129 & 3.330 & 0.146 & 2.034 & 0.048 & 2.466 & 0.796 & 1.414 & 0.409 & 1.821 & 0.592 \\
\hline Lim Non Polynomial & 0.395 & 0.044 & 0.374 & 0.048 & 0.433 & 0.037 & 0.251 & 0.033 & 0.441 & 0.187 & 0.460 & 0.095 \\
\hline Currin Exp & 0.970 & 0.142 & 1.049 & 0.098 & 1.331 & 0.050 & 0.692 & 0.324 & 0.554 & 0.268 & 0.438 & 0.199 \\
\hline Franke & 0.093 & 0.007 & 0.062 & 0.004 & 0.132 & 0.002 & 0.060 & 0.016 & 0.052 & 0.010 & 0.062 & 0.013 \\
\hline Gramacy \& Lee 2008 & 0.058 & 0.002 & 0.069 & 0.001 & 0.074 & 0.001 & 0.040 & 0.006 & 0.035 & 0.008 & 0.035 & 0.006 \\
\hline Sasena & 2.942 & 0.056 & 3.512 & 0.119 & 4.423 & 0.358 & 2.434 & 0.399 & 2.341 & 0.608 & 2.138 & 0.504 \\
\hline Gramacy \& Lee 2012 & 0.426 & 0.067 & 0.527 & 0.097 & 0.508 & 0.034 & 0.456 & 0.071 & 0.458 & 0.073 & 0.471 & 0.127 \\
\hline
\end{tabular}

Table 2: Mean and Standard deviation of RMSE

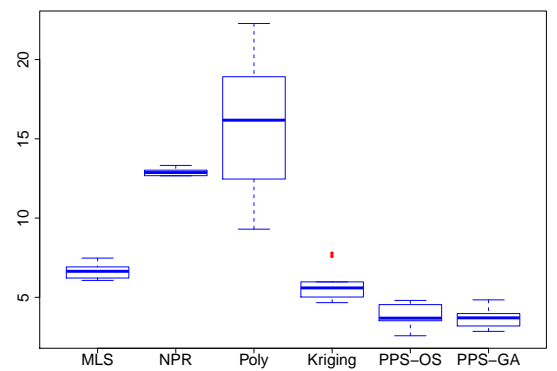

Fig. 2: Wing weight function

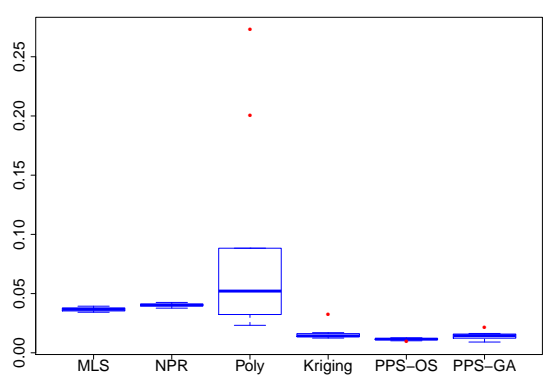

Fig. 5: Piston simulation function

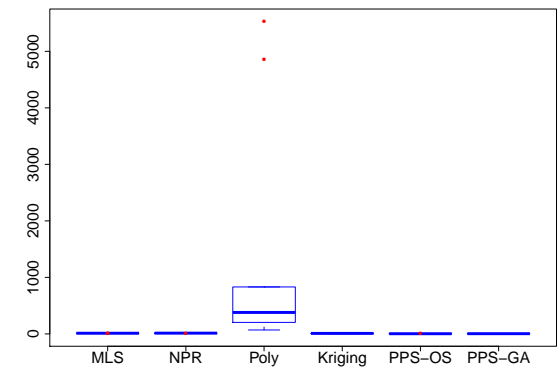

Fig. 3: Borehole function

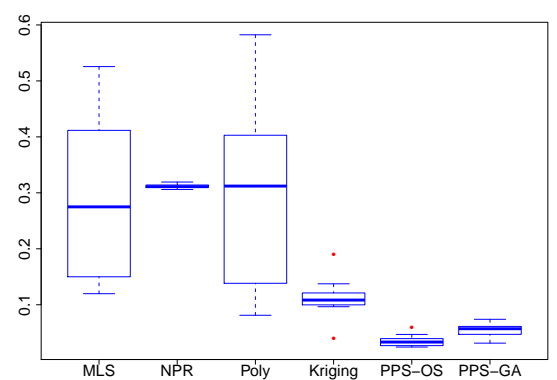

Fig. 6: OTL circuit function

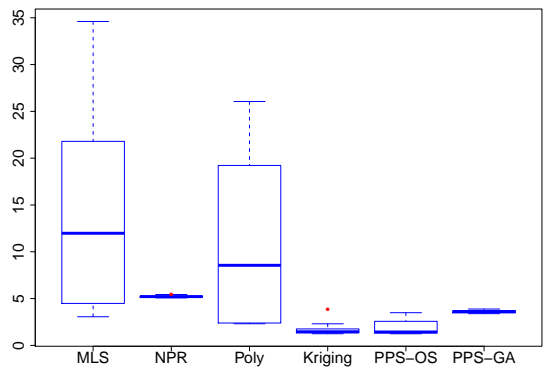

Fig. 4: D \& P (8-Dim) function

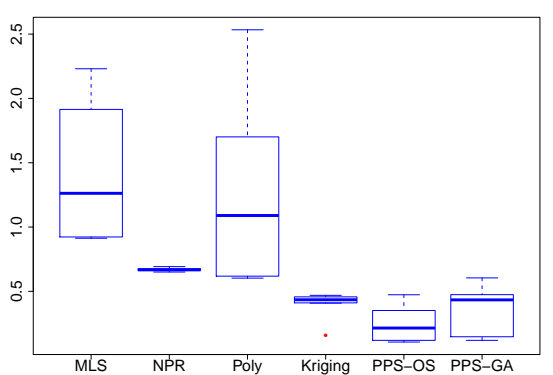

Fig. 7: G \& L 2009 function 


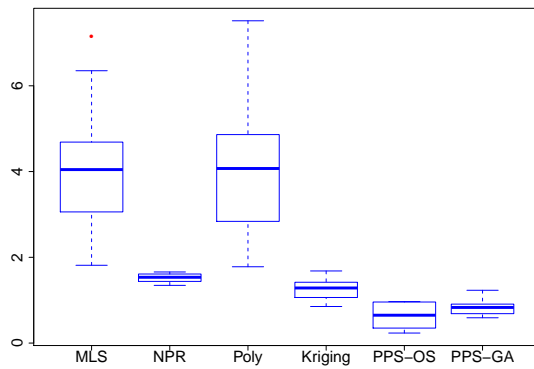

Fig. 8: Friedman function

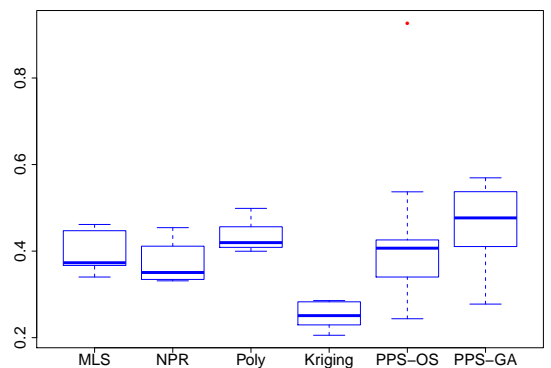

Fig. 11: Lim non-polynomial function

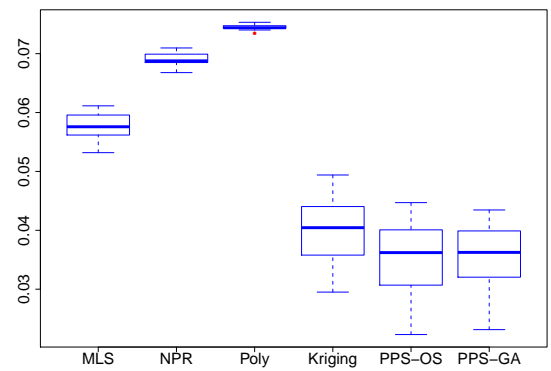

Fig. 14: G \& L (2008) function

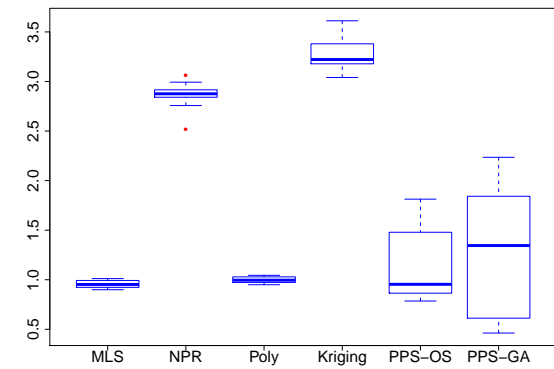

Fig. 9: D \& P exponential function

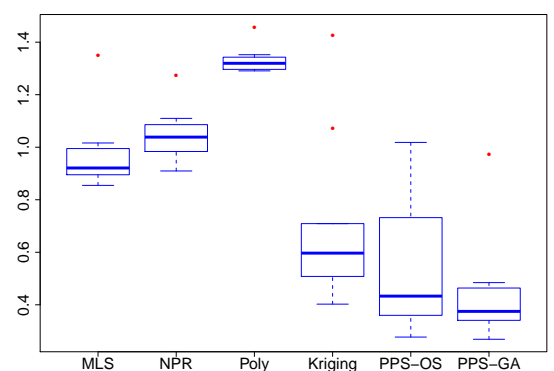

Fig. 12: Currin exponential function

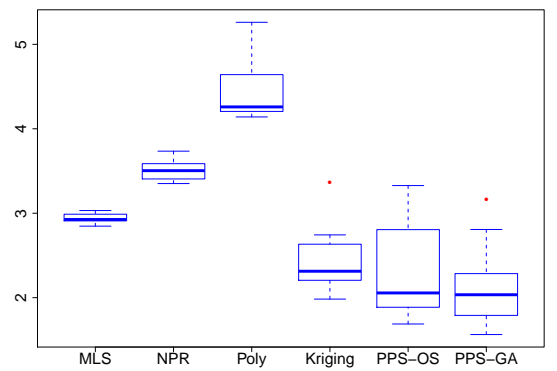

Fig. 15: Sasena function

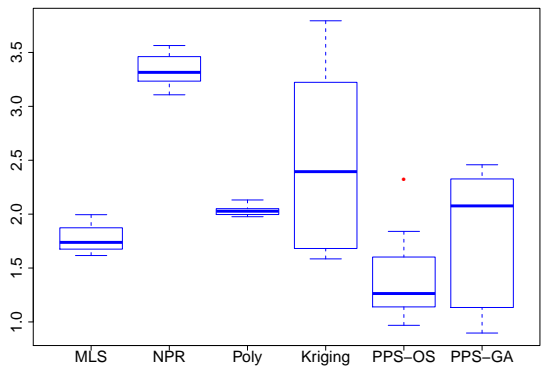

Fig. 10: D \& P curved function

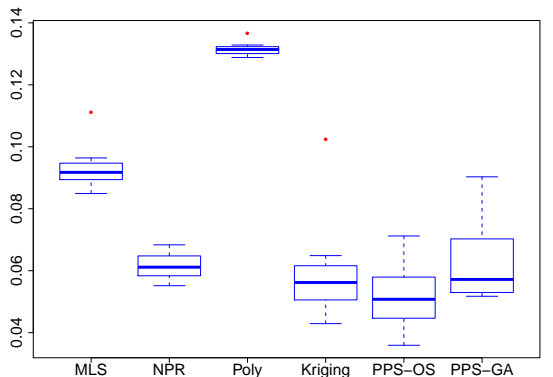

Fig. 13: Franke function

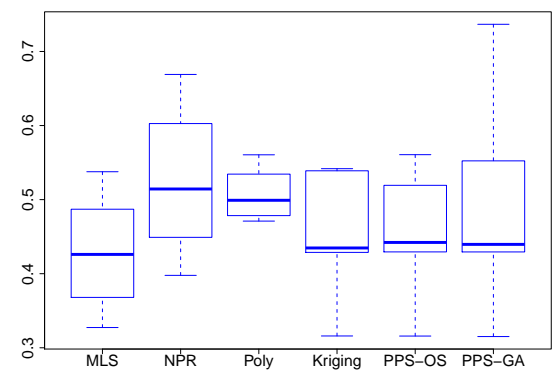

Fig. 16: G \& L 2012 function

\subsection{PPS-based ensembles}

We also use the same test bench to compare the PPS-optimal ensemble, the $O W S$ ensemble and the $P W S$ ensemble with $\alpha=0.05$ and $\beta=-1$. Here, we have at hands 10 surrogate models and we compute the weights by these three different techniques. The results are given in Figure 18. In order to display all the results in the same figure, we have rescaled the values of all the bench functions in $[0,1]$.

Generally, PPS-optimal ensemble give the best result except for the Dette \& Pepelyshev Exp function where the $P W S$ is better and for the Dette \& Pepelyshev 8-Dim function where $O W S$ is better. Moreover, for all the functions, $P P S$-optimal ensemble never has the worst RMSE. This shows the suitability of PPS to construct ensembles.

\subsection{On the choice of $\alpha, \beta$ and $\gamma$}

Further, recall that the PPS criterion is composed of 3 components: MSE, CV and Pen. It is then useful to understand the role played by each component in the performances. To do so, we compared the performance of the algorithms based on the PPS (PPS-GA and PPS-OS) to same algorithms in which we replaced the selection criterion by only one or a sum of two components of the PPS. Therefore, the entire set of tested algorithms are:

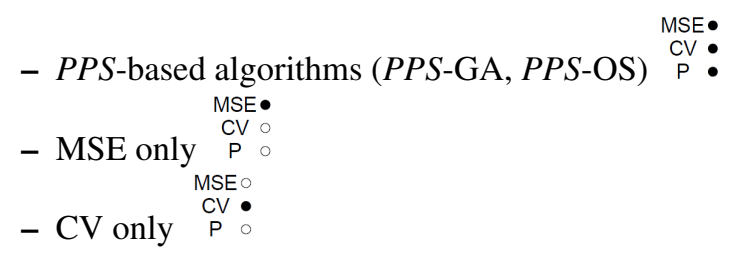




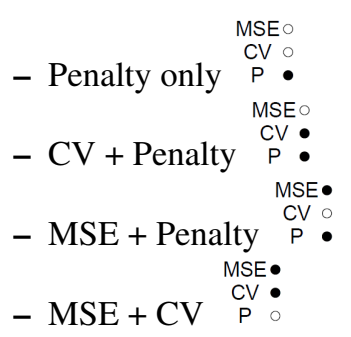

Here, we used LRM as penalty because it gives a reasonable prediction quality when used as a selection criterion. BEF cannot be used in this context. It is only a penalty that favors monomials and constant functions regardless of the output value. We display in Figures $19,20,21$ and 22 the results of the comparison between the different algorithms for some functions of Table 11. Currin function, Sasena function, Dette \& Pepelyshev curved function and Dette \& Pepelyshev 8-dim function. For each function, 10 different maximin LHS design are generated of size $N=10 d$ where $d$ is the space dimension.

For these functions, we can notice how the different components of the PPS act together to select a convenient surrogate model in different scenarios. In fact, the results highlight the effect of each component. Obviously, neither a single criterion nor any combination of two criteria is better than PPS in all the cases. This is due to:
- Any interpolating surrogate model is MSE-optimal. It is a misleading criterion to the overall errors.

- CV is a convenient estimate of the predictive capabilities. But, it is a pessimistic one.

We also study the choice of the values of the parameters of the PPS on the benchmark. We used ten surrogate models and we computed the sum of RMSE for each value of $\beta$ and $\gamma, \alpha$ being fixed to 1 . Let $\left(\beta^{\star}, \gamma^{\star}\right)$ denotes the global minimum. We display the contour plot of the sum of mean square errors (MSE) in Figure 17. Notice that the proposed values of Section $2(1,0.5,0.25)$, are close to the optimum. Further, they give better sum of MSE error 0.3760 if compared to $\left(\beta^{\star}, \gamma^{\star}\right)$ that leads to 0.3771 . We display also the errors of the ensembles using these two parameters in Appendix A. Notice that the prediction errors are close.

\subsection{On the relevance of ensembles}

Finally, we display in Figure 23 the number of surrogate models in the selected surrogate of PPS-GA. We notice that the algorithm selects generally a $P P S$-optimal ensemble. This is due in part to the PPS suitability to ensemble construction and it shows that aggregations are relevant in metamodel se-

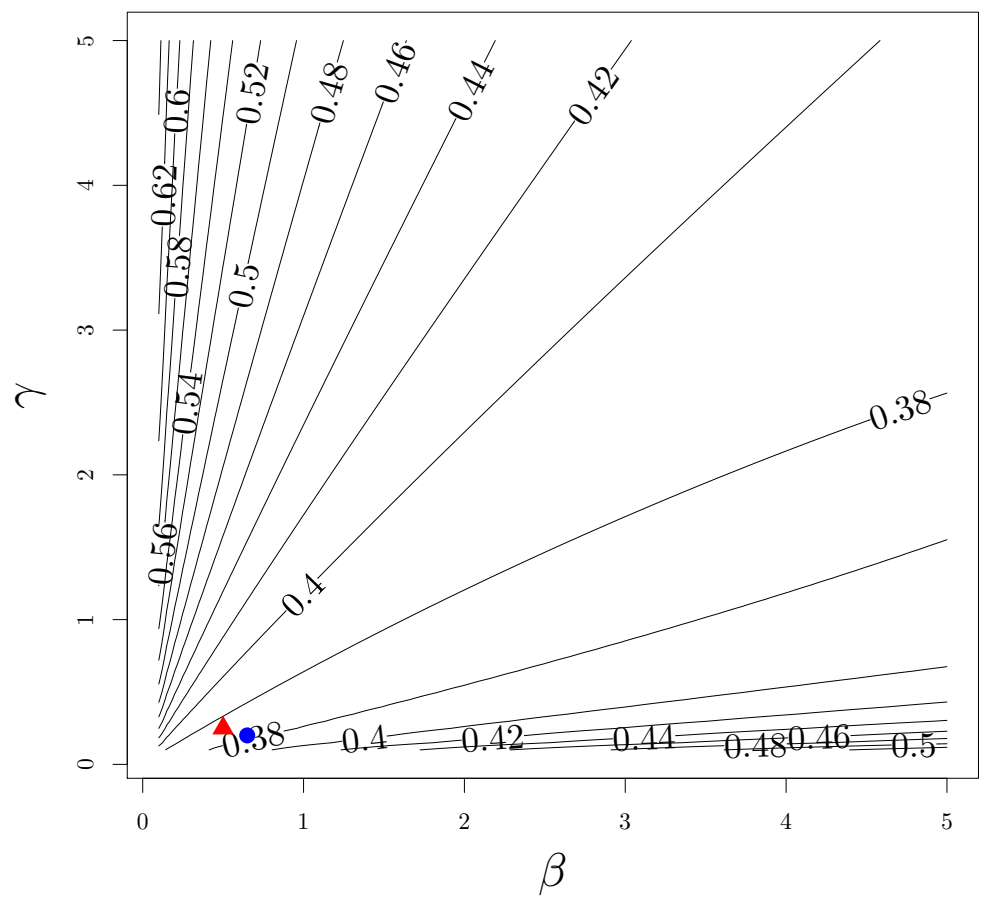

Fig. 17: Contour plot of the sum of scaled MSE of 150 test functions $(15 \times 10$ repetitions), Blue circle: optimum of sum of RMSE, Red triangle: our proposed value. 


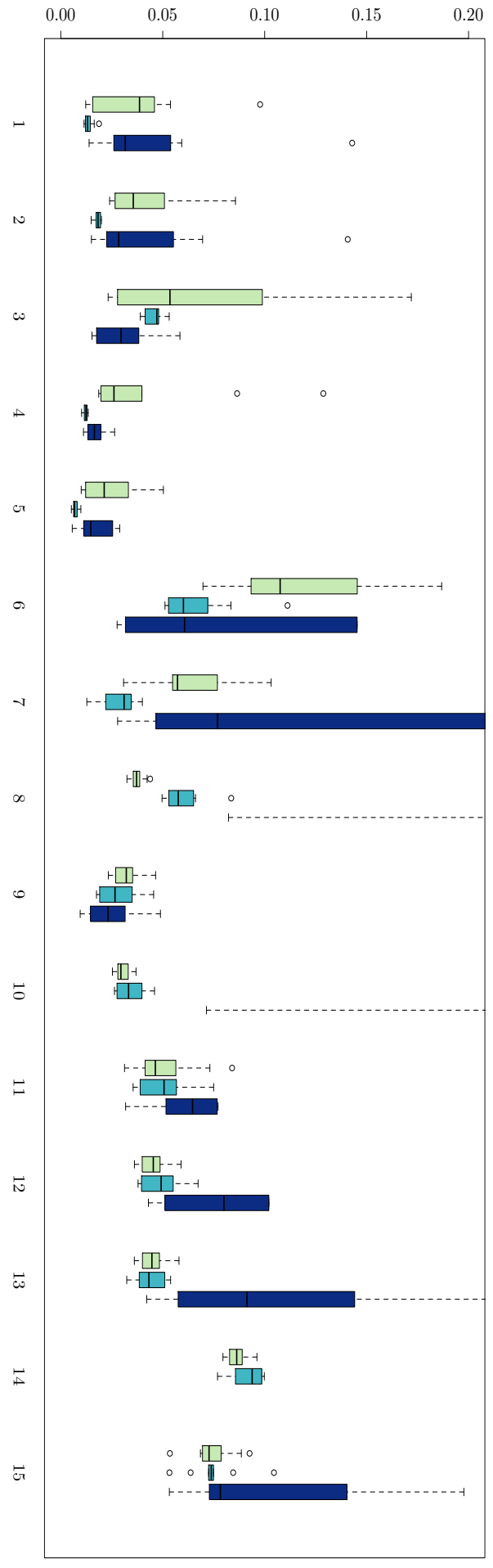

Fig. 18: For each function: Left: $P W S$ method in light green. Middle: PPS-optimal ensemble in light blue. Right: $O W S$ ensemble in dark blue. The function number is as in Table (1) lection. This shows the usefulness of the ensemble approach.

\subsection{Computational cost}

We give in Table 3, the quantiles and the sum of the computing time of all the 150 benchmark functions. It is expected that the selection methods needs more time than individual surrogates. We can notice also that $P P S$-GA is computationally more expensive than PPS-OS. This is due to the cost of exploration. Finally, notice that these values are negligible compared to the computing time of one complex simulation.

\begin{tabular}{rrrrrrr}
\hline & MLS & NPR & Poly & Kriging & PPS-OS & PPS-GA \\
\hline $0 \%$ & 0.000 & 0.000 & 0.000 & 0.000 & 0.012 & 0.386 \\
$25 \%$ & 0.000 & 0.001 & 0.000 & 0.001 & 0.065 & 0.933 \\
$50 \%$ & 0.000 & 0.001 & 0.000 & 0.014 & 0.336 & 1.769 \\
$75 \%$ & 0.000 & 0.003 & 0.003 & 0.068 & 0.886 & 2.865 \\
$100 \%$ & 0.001 & 0.027 & 0.022 & 0.136 & 1.884 & 5.055 \\
Sum & 0.007 & 0.394 & 0.406 & 5.398 & 79.758 & 297.509 \\
\hline
\end{tabular}

Table 3: Elapsed time in seconds to construct each surrogate model

\section{Conclusion}

In this paper, we propose a new selection criterion called the penalized predictive score. PPS can be computed for all the types of surrogate models. By construction, PPS is especially suitable for functions that have specific characteristics such as regularity and smoothness. Generally these characteristics are implicitly expected with the meta-modeling framework. We showed also that it enables the construction of relevant ensembles. The PPS-optimal ensemble are easily computed and avoid over-fitting.

We study also two surrogate model selection schemes based on the PPS. The first one compute the PPS-optimal ensemble rather than selecting one surrogate model. The second one is based on a evolutionary framework that enables the exploration of the space of surrogate models. Tests shows that the proposed algorithms give very good results. It remains important to notice that this algorithm does not necessarily give an accurate approximation in all the cases. For instance, the algorithm will fail if we use a small amount of observations for a highly nonlinear behavior. It aims at selecting the best surrogate among the possible choices. Assessing the level of confidence of a prediction is left for future research.

Acknowledgements We gratefully thank Olivier Roustant and Fabrice Gamboa for the help in writing this paper and for their valuable 


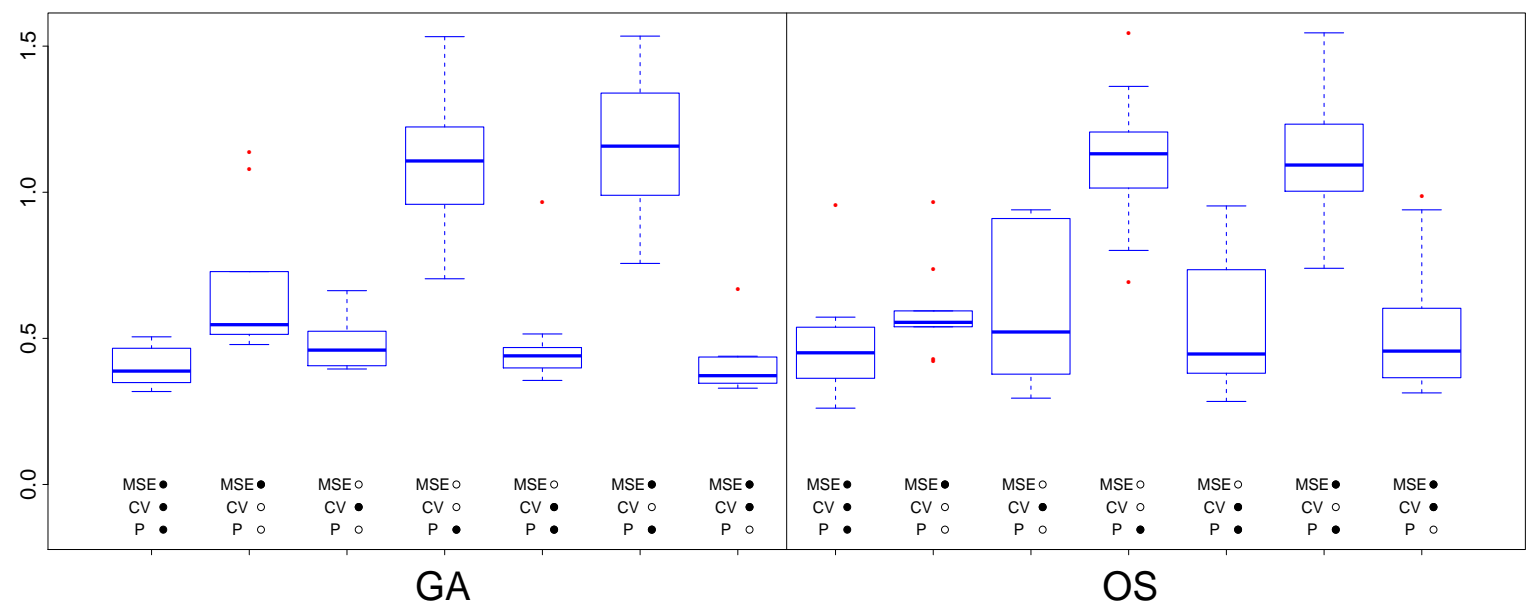

Fig. 19: Several selection criteria: Currin function

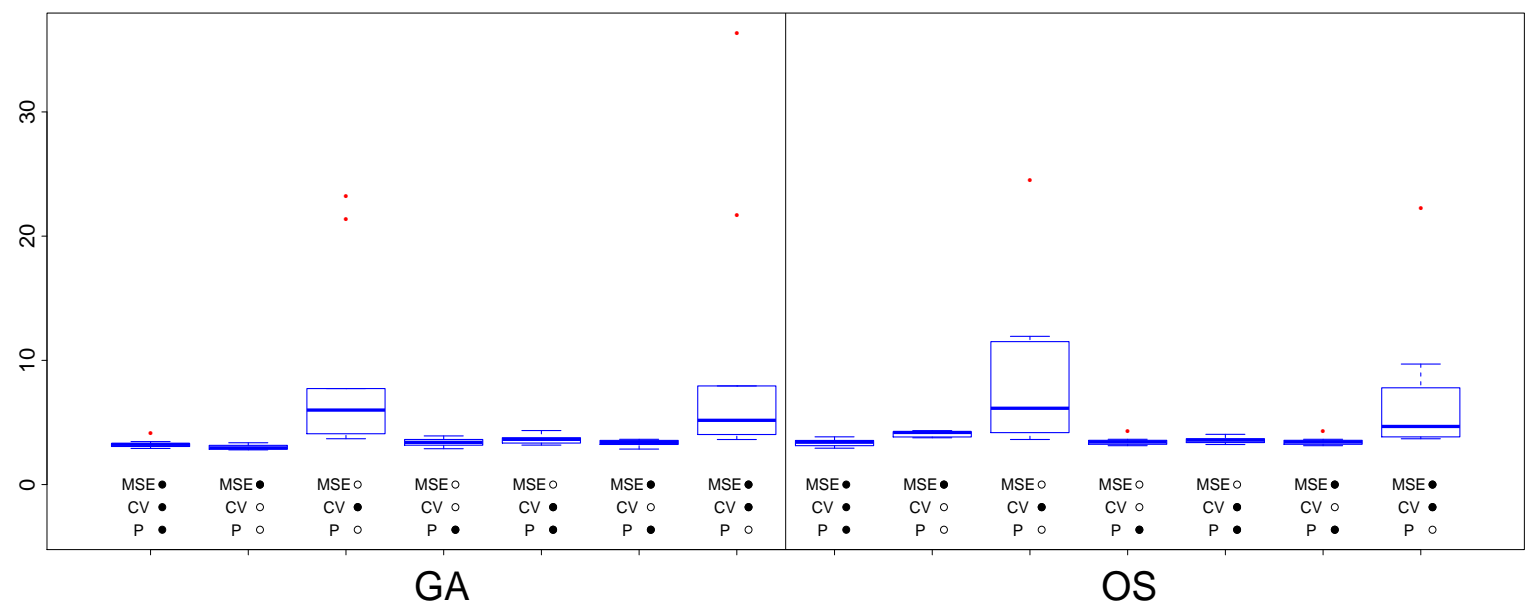

Fig. 20: Several selection criteria: Sasena function

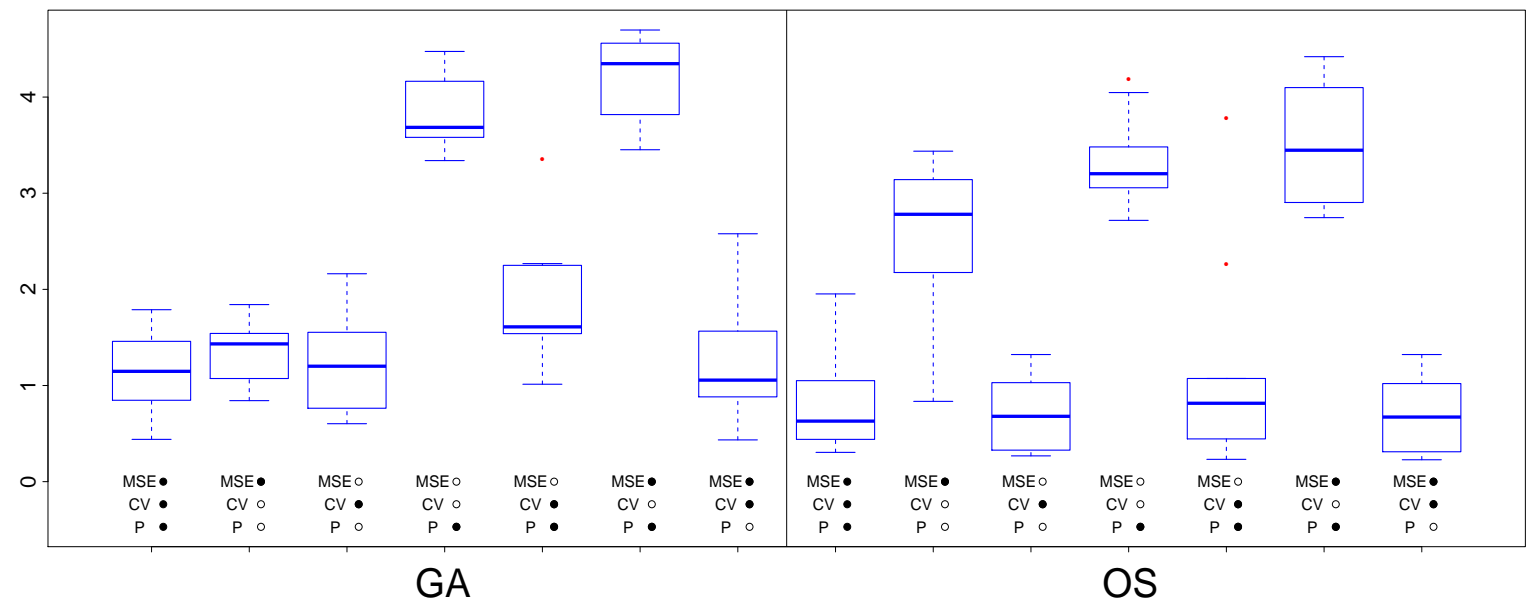

Fig. 21: Several selection criteria: D\&P curved function 


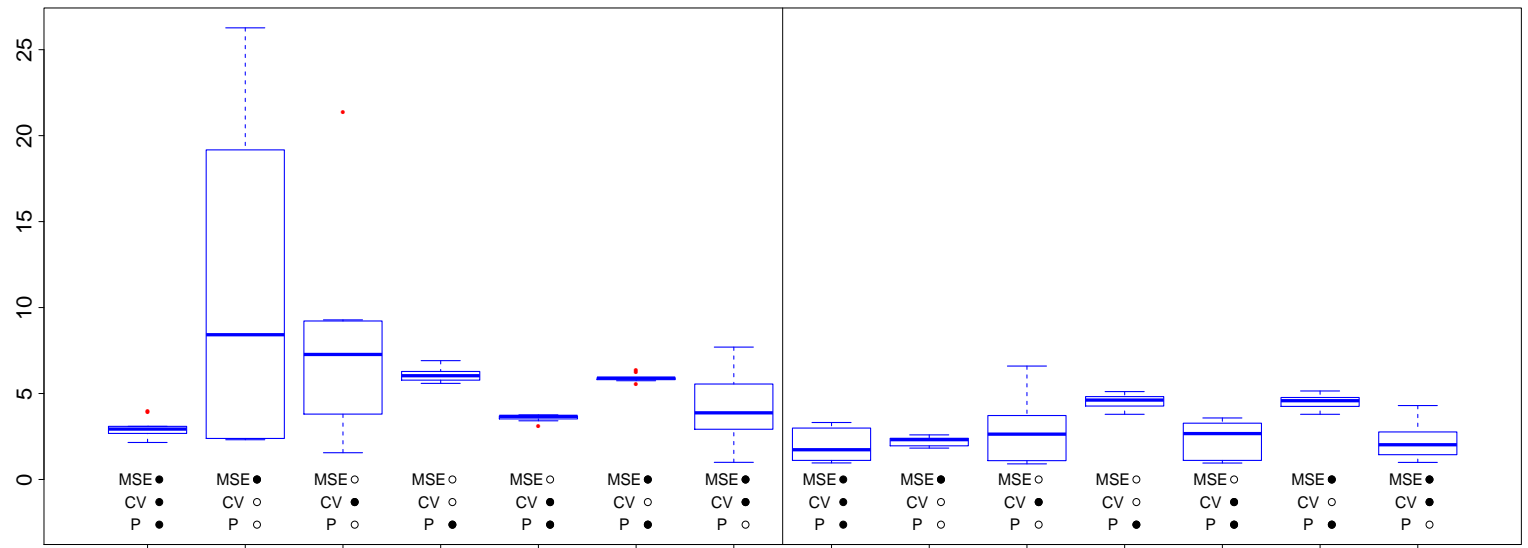

GA

OS

Fig. 22: Several selection criteria: D\&P 8-dim function

remarks. We also warmly thank two anonymous reviewers for their valuable comments.

\section{A Appendix: Comparison between the proposed PPS parameters and the optimal according to the sum of RMSE}

\section{B Appendix: Test functions}

The equations and the input parameter space of the functions of Table 1 are defined below:

1/ Wing weight function:

Parameters: $S_{w} \in[150,200], W_{f w} \in[220,300], A \in[6,10]$, $\gamma \in[-10,10], q \in[16,45], \lambda \in[0.5,1], t_{c} \in[0.08,0.18]$, $N_{z} \in[2.5,6], W_{d g} \in[1700,2500], W_{p} \in[0.025,0.08]$

For $\mathbf{x}=\left(S_{w}, W_{f w} A, \gamma, q, \lambda, t_{c}, N_{z}, W_{d g}, W_{p}\right)$

$f_{1}(\mathbf{x})=0.036 S_{w}^{0.758} W_{f w}^{0.758}\left(\frac{A}{\cos ^{2}(\gamma)}\right)^{0.6} q^{0.006} \lambda^{0.04}$

$$
\left(\frac{100 t_{c}}{\cos (\gamma)}\right)^{-0.3}\left(N_{z} W_{d g}\right)^{0.49}+S_{w} W_{p}
$$

\section{2/ Borehole function:}

Parameters: $r_{w} \in[0.05,0.15], r \in[100,50000]$, $T_{u} \in[63070,115600], H_{u} \in[990,1110], T_{l} \in[63.1,116]$, $H_{l} \in[700,820], L \in[1120,1680], K_{w} \in[9855,12045]$

For $\mathbf{x}=\left(r_{w}, r, T_{u}, H_{u}, T_{l}, H_{l}, L, K_{w}\right)$

$f_{2}(\mathbf{x})=\frac{2 \pi T_{u}\left(H_{u}-H_{l}\right)}{\ln \left(\frac{r}{r_{w}}\right)\left(1+\frac{2 L T_{u}}{\ln \left(\frac{r}{r_{w}}\right) r_{w}^{2} K_{w}}+\frac{T_{u}}{T_{l}}\right)}$
3/ Dette \& Pepelyshev (2010a):

Parameters: for all $i=1, \ldots, 8, x_{i} \in[0,1]$

$$
\begin{aligned}
f_{3}(\mathbf{x}) & =4\left(x_{1}-2+8 x_{2}-8 x_{2}^{2}\right)^{2}+\left(3-4 x_{2}\right)^{2} \\
& +16 \sqrt{x_{3}+1}\left(2 x_{3}-1\right)^{2}+\sum_{i=4}^{8} i \ln \left(1+\sum_{j=3}^{i} x_{j}\right)
\end{aligned}
$$

4/ Piston simulation function:

Parameters: $M \in[30,60], S \in[0.005,0.020]$, $V_{0} \in[0.002,0.010], k \in[1,5] \times 10^{3}, P_{0} \in[9,11] \times 10^{4}$, $T_{a} \in[290,296], T_{0} \in[340,360]$

$$
\begin{gathered}
f_{4}(\mathbf{x})=2 \pi \sqrt{\frac{M}{k+S^{2} \frac{P_{0} V_{0}}{T_{0}} \frac{T_{a}}{V^{2}}}} \\
\text { where } V=\frac{S}{2 k}\left(\sqrt{A^{2}+4 k \frac{P_{0} V_{0}}{T_{0}} T_{a}}-A\right) \\
\text { and } A=P_{0} S+19.62 M-\frac{k V_{0}}{S}
\end{gathered}
$$

5/ OTL circuit function:

Parameters: $R_{b 1} \in[50,150], R_{b 2} \in[25,70]$, $R_{f} \in[0.5,3], R_{c 1} \in[1.2,2.5], R_{c 1} \in[0.25,1.2]$, $\beta \in[50,300]$

$$
\begin{aligned}
f_{5}(\mathbf{R}, \beta) & =\frac{\left(\frac{12 R_{b 2}}{R_{b 1}+R_{b 2}}+0.74\right) \beta\left(R_{c 2}+9\right)}{\beta\left(R_{c 2}+9\right)+R_{f}} \\
& +\frac{11.35 R_{f}}{\beta\left(R_{c 2}+9\right)+R_{f}}+\frac{0.75 R_{f} \beta\left(R_{c 2}+9\right)}{\left(\beta\left(R_{c 2}+9\right)+R_{f}\right) R_{c 1}}
\end{aligned}
$$

6/ Gramacy \& Lee (2009) function:

Parameters: for all $i=1, \ldots, 6, x_{i} \in[0,1]$

$f_{6}(\mathbf{x})=\exp \left[\sin \left(\left(0.9\left(x_{1}+0.48\right)\right)^{10}\right)\right]+x_{2} x_{3}+x_{4}$

7/ Friedman function:

Parameters: for all $i=1, \ldots, 5, x_{i} \in[0,1]$

$f_{7}(\mathbf{x})=10 \sin \left(\pi x_{1} x_{2}\right)+20\left(x_{3}-0.5\right)^{2}+10 x_{4}+5 x_{5}$ 


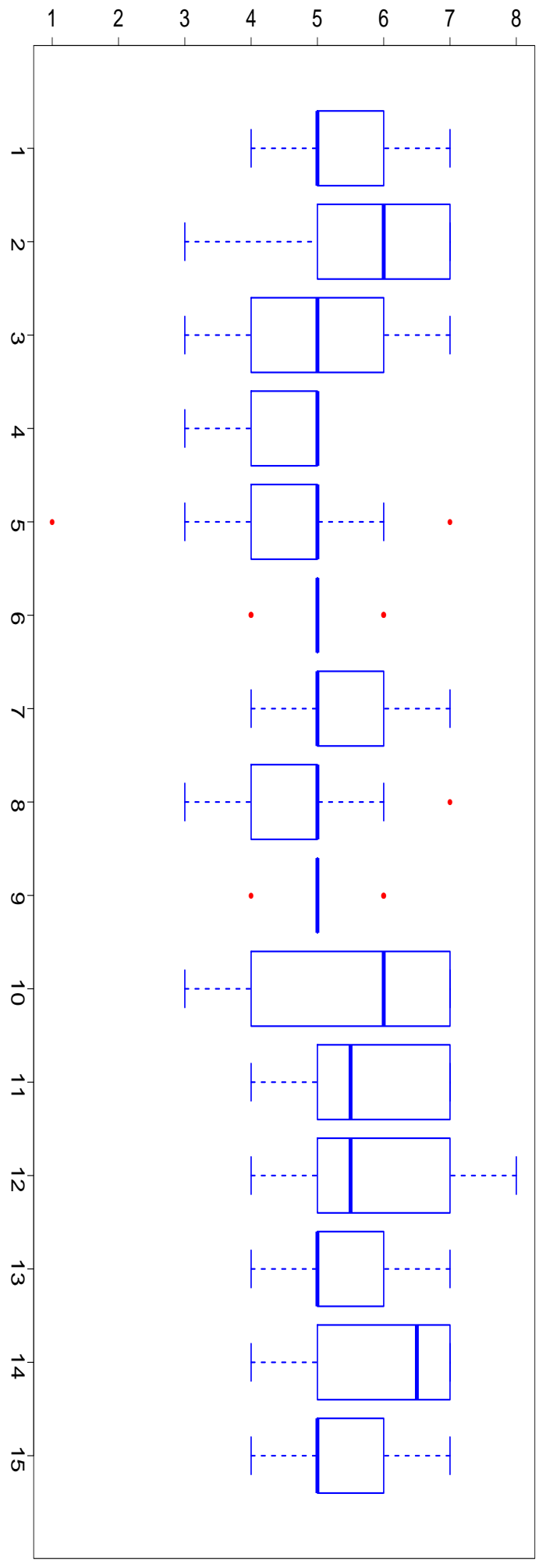

Fig. 23: Number of members in the best ensemble

8/ Dette \& Pepelyshev exponential function:

Parameters: for all $i=1, \ldots, 3, x_{i} \in[0,1]$

$f_{8}(\mathbf{x})=100\left(e^{-2 / x_{1}^{1.75}}+e^{-2 / x_{2}^{1.5}}+e^{-2 / x_{3}^{1.25}}\right)$

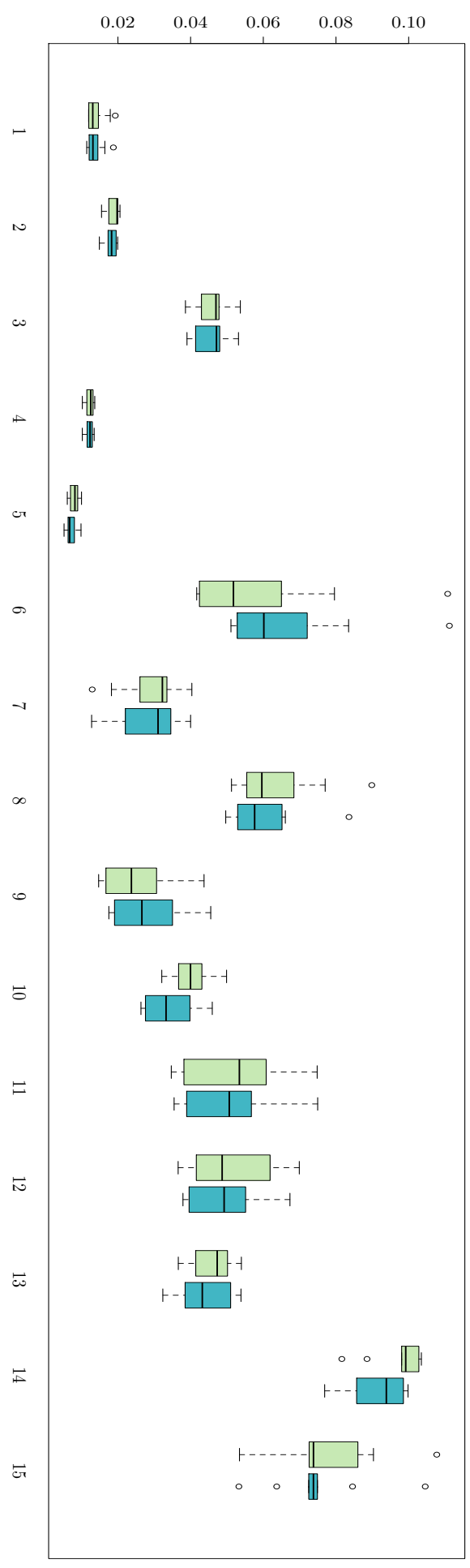

Fig. 24: The scaled RMSE for each PPS-optimal ensemble, For each function: Left: using $(\alpha, \beta, \gamma)=\left(1, \beta^{\star}, \gamma^{\star}\right)$ in light green. Right: using $(\alpha, \beta, \gamma)=(1,0.5,0.25)$ in light blue. The function number is as in Table (1) 
9/ Dette \& Pepelyshev curved function:

Parameters: for all $i=1, \ldots, 3, x_{i} \in[0,1]$

$$
\begin{aligned}
f_{9}(\mathbf{x}) & =4\left(x_{1}-2+8 x_{2}-8 x_{2}^{2}\right)^{2}+\left(3-4 x_{2}\right)^{2} \\
& +16 \sqrt{x_{3}+1}\left(2 x_{3}-1\right)^{2}
\end{aligned}
$$

10/ Lim non-polynomial function:

Parameters: $x_{1}, x_{2} \in[0,1]$

$f_{10}(\mathbf{x})=\frac{1}{6}\left[\left(30+5 x_{1} \sin \left(5 x_{1}\right)\right)\left(4+\exp \left(-5 x_{2}\right)\right)-100\right]$

11/ Currin exponential function:

Parameters: $x_{1}, x_{2} \in[0,1]$

$$
\begin{aligned}
f_{11}(\mathbf{x}) & =\left[1-\exp \left(-\frac{1}{2 x_{2}}\right)\right] \\
& \times \frac{2300 x_{1}^{3}+1900 x_{1}^{2}+2092 x_{1}+60}{100 x_{1}^{3}+500 x_{1}^{2}+4 x_{1}+20}
\end{aligned}
$$

12/ Franke function:

Parameters: $x_{1}, x_{2} \in[0,1]$

$$
\begin{aligned}
f_{12}(\mathbf{x}) & =0.75 \exp \left(-\frac{\left(9 x_{1}-2\right)^{2}+\left(9 x_{2}-2\right)^{2}}{4}\right) \\
& +0.75 \exp \left(-\frac{\left(9 x_{1}+2\right)^{2}}{49}-\frac{9 x_{2}+1}{10}\right) \\
& +0.5 \exp \left(-\frac{\left(9 x_{1}-7\right)^{2}}{4}-\frac{\left(9 x_{2}-3\right)^{2}}{4}\right) \\
& +0.2 \exp \left(-\left(9 x_{1}-4\right)^{2}-\left(9 x_{2}-7\right)^{2}\right)
\end{aligned}
$$

13/ Gramacy \& Lee (2008) function:

Parameters: $x_{1}, x_{2} \in[-2,6]$

$f_{13}(\mathbf{x})=x_{1} \exp \left(-x_{1}^{2}-x_{2}^{2}\right)$

14/ Sasena function:

Parameters: $x_{1}, x_{2} \in[0.0,5]$

$$
\begin{aligned}
f_{14}(\mathbf{x}) & =2+0.01\left(x_{2}-x_{1}^{2}\right)^{2}+\left(1-x_{1}\right)^{2} \\
& +2\left(2-x_{2}\right)^{2}+7 \sin \left(0.5 x_{1}\right) \sin \left(0.7 x_{1} x_{2}\right)
\end{aligned}
$$

15/ Gramacy \& Lee (2012) function:

Parameters: $x \in[0.5,2.5]$

$f_{15}(x)=\frac{\sin (10 \pi x)}{2 x}+(x-1)^{4}$

\section{References}

Acar E, Rais-Rohani M (2009) Ensemble of metamodels with optimized weight factors. Structural and Multidisciplinary Optimization 37(3):279-294

Akaike H (1974) A new look at the statistical model identification. Automatic Control, IEEE Transactions on 19(6):716-723
Arlot S, Celisse A (2010) A survey of cross-validation procedures for model selection. Statist Surv 4:40-79

Chen PW, Wang JY, Lee HM (2004) Model selection of svms using ga approach. In: Neural Networks, 2004. Proceedings. 2004 IEEE International Joint Conference on, IEEE, vol 3, pp 2035-2040

Duchon J (1977) Splines minimizing rotation-invariant semi-norms in sobolev spaces. In: Constructive theory of functions of several variables, Springer, pp 85-100

Efron B, Tibshirani RJ (1993) An introduction to the bootstrap

Forrester AI, Keane AJ (2009) Recent advances in surrogate-based optimization. Progress in Aerospace Sciences 45(1):50-79

Friedman JH (1991) Multivariate adaptive regression splines. The annals of statistics pp 1-67

Goel T, Haftka RT, Shyy W, Queipo NV (2007) Ensemble of surrogates. Structural and Multidisciplinary Optimization 33(3):199-216

Gorissen D, Dhaene T, Turck FD (2009) Evolutionary model type selection for global surrogate modeling. The Journal of Machine Learning Research 10:2039-2078

Hastie T, Tibshirani R, Friedman J (2009) The elements of statistical learning, vol 2. Springer

James G, Witten D, Hastie T, Tibshirani R (2013) An introduction to statistical learning, vol 6. Springer

Kohavi R (1995) A study of cross-validation and bootstrap for accuracy estimation and model selection. In: Proceedings of the 14th International Joint Conference on Artificial Intelligence - Volume 2, Morgan Kaufmann Publishers Inc., IJCAI'95, pp 1137-1143

Lancaster P, Salkauskas K (1981) Surfaces generated by moving least squares methods. Mathematics of computation 37(155):141-158

Lessmann S, Stahlbock R, Crone SF (2006) Genetic algorithms for support vector machine model selection. In The 2006 IEEE International Joint Conference on Neural Network Proceedings, IEEE, pp 3063-3069

Matheron G (1963) Principles of geostatistics. Economic geology 58(8):1246-1266

McKay MD, Beckman RJ, Conover WJ (1979) Comparison of three methods for selecting values of input variables in the analysis of output from a computer code. Technometrics 21(2):239-245

Müller J, Piché R (2011) Mixture surrogate models based on dempster-shafer theory for global optimization problems. Journal of Global Optimization 51(1):79-104

Nguyen H, Couckuyt I, Knockaert L, Dhaene T, Gorissen D, Saeys Y (2011) An alternative approach to avoid overfitting for surrogate models. In: Simulation Conference (WSC), Proceedings of the 2011 Winter, pp 2760-2771

Queipo NV, Haftka RT, Shyy W, Goel T, Vaidyanathan R, Tucker PK (2005) Surrogate-based analysis and optimiza- 
tion. Progress in aerospace sciences 41(1):1-28

Schwarz G, et al (1978) Estimating the dimension of a model. The annals of statistics 6(2):461-464

Shi L, Yang R, Zhu P (2012) A method for selecting surrogate models in crashworthiness optimization. Structural and Multidisciplinary Optimization 46(2):159-170

Smola A, Schlkopf B (2004) A tutorial on support vector regression. Statistics and Computing 14(3):199-222

Stone M (1974) Cross-validatory choice and assessment of statistical predictions. Journal of the Royal Statistical Society Series B (Methodological) pp 111-147

Tomioka S, Nisiyama S, Enoto T (2007) Nonlinear least square regression by adaptive domain method with multiple genetic algorithms. IEEE Transactions on Evolutionary Computation 11(1):1-16

Viana FA, Haftka RT, Steffen V (2009) Multiple surrogates: how cross-validation errors can help us to obtain the best predictor. Structural and Multidisciplinary Optimization 39(4):439-457

Viana FA, Venter G, Balabanov V (2010) An algorithm for fast optimal latin hypercube design of experiments. International journal for numerical methods in engineering 82(2):135-156

Watson DF (1981) Computing the n-dimensional delaunay tessellation with application to voronoi polytopes. The computer journal 24(2):167-172

Zerpa LE, Queipo NV, Pintos S, Salager JL (2005) An optimization methodology of alkaline-surfactant-polymer flooding processes using field scale numerical simulation and multiple surrogates. Journal of Petroleum Science and Engineering 47(3):197-208

Zhang C, Shao H, Li Y (2000) Particle swarm optimisation for evolving artificial neural network. In: Systems, Man, and Cybernetics, 2000 IEEE International Conference on, IEEE, vol 4, pp 2487-2490

Zhou X, Jiang T (2016) Metamodel selection based on stepwise regression. Structural and Multidisciplinary Optimization 54(3):641-657

Zhou XJ, Ma YZ, Li XF (2011) Ensemble of surrogates with recursive arithmetic average. Structural and Multidisciplinary Optimization 44(5):651-671 